\title{
Maximum brightness of linac-driven electron beams in the presence of collective effects
}

\author{
S. Di Mitri \\ Elettra-Sincrotrone Trieste S.C.p.A., 34149 Basovizza, Trieste, Italy
}

(Received 7 December 2012; published 7 May 2013)

\begin{abstract}
Linear accelerators capable of delivering high brightness electron beams are essential components of a number of research tools, such as free electron lasers (FELs) and elementary particle colliders. In these facilities the charge density is high enough to drive undesirable collective effects (wakefields) that may increase the beam emittance relative to the injection level, eventually degrading the nominal brightness. We formulate a limit on the final electron beam brightness, imposed by the interplay of geometric transverse wakefield in accelerating structures and coherent synchrotron radiation in energy dispersive regions. Numerous experimental data of vacuum ultraviolet and x-ray FEL drivers validate our model. This is then used to show that a normalized brightness of $\sim 10^{16} \mathrm{~A} / \mathrm{m}^{2}$, promised so far by ultralow charge beams $(\sim 1-10 \mathrm{pC})$, can in fact be reached with a $100 \mathrm{pC}$ charge beam in the $1.2 \mathrm{GeV}$ FERMI@Elettra accelerator, with the existing machine configuration.
\end{abstract}

DOI: 10.1103/PhysRevSTAB.16.050701

PACS numbers: 29.20.Ej, 29.27.Bd

\section{INTRODUCTION}

State-of-the-art linear accelerators for x-ray free electron lasers (FELs) and particle colliders generate electron beams with high brightness and small longitudinal emittance [1,2]. High brightness is required in order to achieve a high luminosity in colliders and short radiation gain length in FELs. In these facilities the beam charge density is high enough to drive collective effects (wakefields) that, notwithstanding the high beam rigidity at energies up to the $\mathrm{GeV}$ range, may increase the emittance relative to its injection level. Geometric transverse wakefield (GTW) in the radiofrequency linear accelerator (rf linac) and emission of coherent synchrotron radiation (CSR) in energy dispersive regions (such as transfer lines or magnetic bunch length compressors) are the dominant collective processes responsible for diluting the beam transverse emittance (see, e.g., $[3,4]$ and references therein), with consequent reduction of the brightness. The (normalized) brightness is hereafter defined as the ratio of the bunch final peak current over the product of the two transverse (normalized) emittances [5].

In this article we formulate a limit on the maximum brightness of an ultrarelativistic electron beam achievable in a single-pass linac when collective effects are part of the dynamics. The limit is determined by the interplay of the dispersion strength (e.g., bunch length compression factor) with the linac GTW and CSR effect on the transverse emittance. We validate our model by means of a systematic comparison of theoretical predictions with

Published by the American Physical Society under the terms of the Creative Commons Attribution 3.0 License. Further distribution of this work must maintain attribution to the author(s) and the published article's title, journal citation, and DOI. experimental data of existing vacuum ultraviolet and x-ray FEL drivers. We show that the effects of GTW and CSR on the final beam quality, which has traditionally been treated separately in the archival literature, are coupled by the variation of the bunch length along the beam line. Hence, GTW and CSR should be considered simultaneously during machine design.

The choice of machine configuration, i.e., bunch charge, initial bunch length, compression factor, and so on-hereafter referred to as the machine "working point"-is typically the result of an iterative process which relies on particle tracking studies such as startto-end, time-dependent simulations $[3,6]$ and multiobjective optimization algorithms [7,8]. Without aiming to replace these powerful and sophisticated techniques, the analysis proposed in this paper purports to be useful as an exploratory and fast tool to maximize the beam brightness. It is worth mentioning that, in principle, the brightness can always be improved by brute force, e.g., increasing the beam energy, thus with a direct impact on the accelerator size and cost. Our analysis, instead, aims to maximize the brightness by improving the accelerator performance once the beam line is given, thus with no additional costs.

In the following, well-known expressions for geometric and CSR wake functions are used for modeling the beam dynamics in order to: (i) evaluate the brightness degradation due to the collective effects, (ii) recognize the dominant source of emittance dilution, and (iii) maximize the brightness with a proper choice of the working point. The analytical expression of the GTW is that derived under the steady-state, periodic structure approximation $[9,10]$. As for CSR, we apply the analytical model of steady-state regime of emission by a short bunch in a long magnet [11]. We show that these assumptions, generally true in real facilities, allow a 
computation of the final brightness in agreement with values measured in LCLS [12] and FERMI@Elettra FEL [13].

\section{THEORETICAL MODEL}

The present understanding of CSR effects on ultrarelativistic beam transverse emittance may be traced back to [11,14-21]. In summary, CSR fields affect the electron transverse motion both with their direct radial forces and by changing the particle energy in the dispersive line. The latter process typically dominates, wherein a particle starts a betatron oscillation around a new reference trajectory, thus increasing its CourantSnyder (C-S) invariant [22]. The synchrotron radiation emission is coherent for wavelengths comparable to the electron bunch length and it induces a variation of the particle energy that is correlated with the longitudinal coordinate along the bunch. This correlation translates into a projected emittance growth. In magnetic compressors or short dispersive lines, the CSR effect is commonly mitigated by shrinking the optical $\beta$ function down to the meter level. This is done in order to make the beam angular divergence locally much larger than the CSR kick, so that it has little or no impact on the transverse particle distribution. By using the beam matrix formalism, as suggested in [23], one finds that the CSR-induced normalized emittance growth (in the bending plane) is

$$
\begin{aligned}
\Delta \gamma_{0} \varepsilon_{i, 0} & \cong \gamma_{0} \varepsilon_{i, 0}\left[\sqrt{1+\frac{\hat{\beta} \theta^{2} \sigma_{\delta, \mathrm{CSR}}^{2}}{\varepsilon_{i, 0}}}-1\right] \\
& \equiv \gamma_{0} \varepsilon_{i, 0}\left[\sqrt{1+X_{\mathrm{CSR}}}-1\right]
\end{aligned}
$$

where $\gamma_{0}$ and $\varepsilon_{i, 0}$ are, respectively, the relativistic Lorentz factor and the unperturbed geometric emittance at the dispersive insertion, $\theta$ is bending angle, $\hat{\beta}$ is the minimum value of $\beta$ in the insertion, and $\sigma_{\delta, \operatorname{CSR}}$ is the CSR-induced rms energy spread relative to the beam mean energy. The latter is [11]

$$
\sigma_{\delta, \mathrm{CSR}}=0.2459 \frac{r_{e}}{e} \frac{Q}{\gamma}\left(\frac{l \theta^{2} C^{4}}{\sigma_{z, 0}^{4}}\right)^{1 / 3}
$$

where we introduced the electron classical radius $r_{e}$ and the electron charge $e, Q$ is the bunch total charge, $l$ the dipole magnet length, $\sigma_{z, 0}$ the initial rms bunch length, and $C$ the (linear) compression factor, i.e., the ratio of the initial over the final bunch length:

$$
C=\frac{\sigma_{z, 0}}{\sigma_{z, f}}=\frac{1}{1+h R_{56}} \approx \frac{1}{1+\frac{\sigma_{\delta, 0}}{\sigma_{z, 0}} R_{56}} .
$$

Here $\sigma_{\delta, 0}$ is the relative energy spread imparted to the beam by the upstream rf off-crest acceleration. The longitudinal transport matrix element $R_{56}$ is determined by the dispersive insertion geometry and is, for a four dipoles, achromatic and symmetric chicane with $\theta \ll 1$, $R_{56} \cong-2 \theta^{2}\left(L_{12}+\frac{2}{3} l\right)$, where $L_{12}$ is the drift length between the two outer bending magnets. In our convention, a negative $R_{56}$ compresses the bunch duration if the linearly correlated energy chirp $h$ is positive.

The single-bunch transverse wakefield instability [24], which happens when the bunch travels at a distance $\Delta$ from the linac electric axis, generates a displacement of the trailing particles with respect to the bunch head; this displacement is correlated with the longitudinal coordinate along the bunch. The trailing particles start a betatron oscillation around a new dispersive trajectory, therefore increasing their C-S invariant. Such an effect can be removed by finding a "golden" trajectory which makes the wakefield's kicks cancel each other (emittance bumps) [25-27]. In analogy with the CSR case, the transverse emittance dilution at the linac end is [26]

$$
\begin{aligned}
\Delta \gamma_{f} \varepsilon_{f, 0} & \cong \gamma_{f} \varepsilon_{f, 0}\left[\sqrt{1+2\left(\frac{\pi r_{e}}{Z_{0} c e}\right)^{2} \frac{Q^{2} \bar{W}_{\perp}^{2} \Delta^{2} L_{\mathrm{FODO}} L_{\mathrm{tot}} \bar{\beta}}{\gamma_{f} \varepsilon_{f, 0} \sqrt{\gamma_{0} \gamma_{f}}}}-1\right] \\
& \equiv \gamma_{f} \varepsilon_{f, 0}\left[\sqrt{1+X_{W}}-1\right]
\end{aligned}
$$

where $Z_{0}=377 \Omega$ is the vacuum impedance, $c$ is the speed of light in vacuum, $\gamma_{f}$ and $\varepsilon_{f, 0}$ are, respectively, the relativistic Lorentz factor and the unperturbed geometric emittance at the linac end, $\bar{\beta}$ the average betatron function along the linac of length $L_{\text {tot }}$, whose accelerating structures have all length $L_{\mathrm{FODO}} / 2, \Delta$ is the rms offset of the randomly displaced accelerating structures, and $\bar{W}_{\perp}$ is the geometric transverse wake function per unit length computed at $z=2 \sigma_{z}$. The short-range transverse wake function of a cylindrical axis-symmetric structure is given by [9]

$$
W_{\perp}(z)=A\left[1-\left(1+\sqrt{\frac{z}{s_{1}}}\right) \exp \left(-\sqrt{\frac{z}{s_{1}}}\right)\right]
$$

where $A$ and $s_{1}$ depend on the geometry of the accelerating structure's inner cells. For an $S$-band linac, we typically have $s_{1}$ in the range $0.5-0.7 \mathrm{~mm}$, and $A$ in the range $10^{3}-10^{4} \mathrm{~V} / \mathrm{pC} / \mathrm{m}^{2}[4,9]$. If we assume that CSR and GTW kicks are uncorrelated and that the bunch length compression is in one plane only, the final normalized beam brightness is 


$$
\begin{aligned}
B_{n, f} & \equiv \frac{I}{\gamma_{f}^{2} \varepsilon_{x, f} \varepsilon_{y, f}} \\
& =\frac{C I_{0}}{\left(\gamma_{0} \varepsilon_{i, 0}\right)^{2} \sqrt{\left(1+X_{W}\right)\left(1+X_{\mathrm{CSR}}+X_{W}\right)}} \\
& \equiv \frac{C B_{n, 0}}{\sqrt{\left(1+X_{W}\right)\left(1+X_{\mathrm{CSR}}+X_{W}\right)}}
\end{aligned}
$$

where $\gamma_{f} \varepsilon_{f, 0}=\gamma_{0} \varepsilon_{i, 0}$ is a constant of motion and we have assumed for simplicity identical unperturbed normalized emittances in the transverse planes. The final normalized brightness $B_{n, f}$ is linearly proportional to the initial normalized brightness $B_{n, 0}$, as it comes from the injector [28], times the compression factor $C$. We define the brightness efficiency as $B_{n, f} / C B_{n, 0}$. We note that Eq. (6) does not include any free parameter to fit experimental data.

\section{EXPERIMENTAL VALIDATION OF THE THEORETICAL BRIGHTNESS}

Table I shows the agreement between the brightness predicted by Eq. (6) and the experimental value measured at the end of the LCLS and the FERMI linacs. The beam lines of these two facilities are sketched in Fig. 1. The experimental brightness is the ratio of the measured peak current and the product of the measured normalized emittances. We refer to data in $[29,30]$ for LCLS and to [31,32] for FERMI-250 pC. The data for the
FERMI-500 pC case were determined with methods described in $[23,31,33]$.

Figure 2 shows the theoretical contribution of CSR and GTW to the brightness of a $250 \mathrm{pC}$ charge beam as a function of the compression factor in LCLS and in FERMI. The vertical dashed line identifies the actual working point of the two facilities (see also Table I). In spite of the same charge, the LCLS beam dynamics is predicted to be dominated by the CSR instability, while the GTW instability dominates in FERMI. The FERMI brightness is in fact dominated by the $\sim 20$ times larger GTW amplitude, whose magnitude is in turn determined by the small $(\sim 5 \mathrm{~mm})$ accelerating cells' iris radius. It is comparable to wake amplitudes in $X$-band structures [34], although proposed $X$-band facilities are planned to run at much higher energies and with lower charge than FERMI, and will thus be less sensitive to collective effects $[35,36]$.

The theoretical brightness was further compared to the experimental one in FERMI over a $250-500$ pC charge range, and for different bunch lengths. The result is in Fig. 3. According to plots similar to Fig. 2 (not shown), the GTW is expected to dominate over the full range of charges. This is depicted by the dashed line that describes the theoretical brightness dependence on charge $Q$ and compression factor $C$ when only GTW is considered. The uncertainty on the theoretical brightness is dominated by the uncertainty on the linac misalignment $(\Delta)$ and the focusing properties $(\bar{\beta})$. Figure 4 shows the

TABLE I. LCLS and FERMI electron beam and machine parameters. The peak current $I_{0}$ is defined as $Q c$ divided by $3.5 \sigma_{z, 0}$, where

\begin{tabular}{|c|c|c|c|c|c|}
\hline Parameter & LCLS-250 & LCLS-1000 & FERMI-250 & FERMI-500 & Units \\
\hline$Q$ & 250 & 1000 & 250 & 500 & $\mathrm{pC}$ \\
\hline$\sigma_{t, 0}$ & 2.5 & 2.8 & 2.4 & 2.8 & ps \\
\hline$I_{0}$ & 30 & 100 & 30 & 50 & $\mathrm{~A}$ \\
\hline C & $5 \times 20$ & $5 \times 7$ & 7 & 12 & $\cdots$ \\
\hline$\gamma_{0}$ & 490 & 490 & 590 & 550 & $\cdots$ \\
\hline$\gamma_{f}$ & 26420 & 26420 & 2450 & 2450 & $\cdots$ \\
\hline$L_{\mathrm{FODO}}, L_{\mathrm{tot}}$ & 6,790 & 6,790 & 12,45 & 12,45 & $\mathrm{~m}$ \\
\hline $\bar{\beta}$ & 35 & 35 & 25 & 25 & $\mathrm{~m}$ \\
\hline$\hat{\beta}$ & 3 & 3 & 3 & 3 & $\mathrm{~m}$ \\
\hline$\Delta$ & 200 & 200 & 200 & 100 & $\mu \mathrm{m}$ \\
\hline$W_{\perp 0}$ & $1 \times 10^{16}$ & $1 \times 10^{16}$ & $17 \times 10^{16}$ & $17 \times 10^{16}$ & $\mathrm{~V} / \mathrm{C} / \mathrm{m}^{2}$ \\
\hline$\varepsilon_{n 0}$ & 0.7 & 1.2 & 1.0 & 1.0 & $\mu \mathrm{m} \mathrm{rad}$ \\
\hline$\varepsilon_{n f}$ & 1.0 & 1.5 & 2.3 & 2.0 & $\mu \mathrm{m} \mathrm{rad}$ \\
\hline$C B_{n, 0}$ & $5.9 \times 10^{15}$ & $2.4 \times 10^{15}$ & $2.1 \times 10^{14}$ & $6.1 \times 10^{14}$ & $\mathrm{~A} / \mathrm{m}^{2}$ \\
\hline$B_{n, f}($ exp.) & $3.0 \times 10^{15}$ & $\leq 1.5 \times 10^{15}$ & $0.4 \times 10^{14}$ & $1.5 \times 10^{14}$ & $\mathrm{~A} / \mathrm{m}^{2}$ \\
\hline$B_{n, f}$ [Eq. (6)] & $3.3 \times 10^{15}$ & $1.6 \times 10^{15}$ & $0.4 \times 10^{14}$ & $1.7 \times 10^{14}$ & $\mathrm{~A} / \mathrm{m}^{2}$ \\
\hline
\end{tabular}
$Q$ is the beam charge, $c$ is the light speed in vacuum, and $\sigma_{z, 0}$ is the rms bunch length. The normalized emittance is the geometric mean of the horizontal and the vertical ones. 


\section{LCLS LINAC}

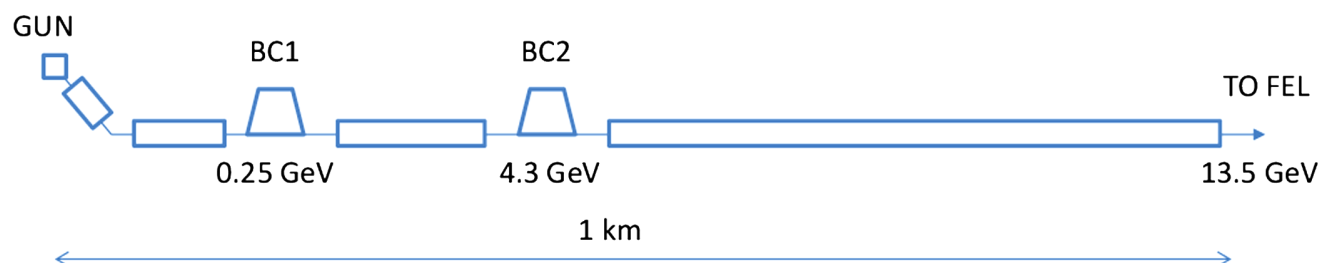

FERMI LINAC

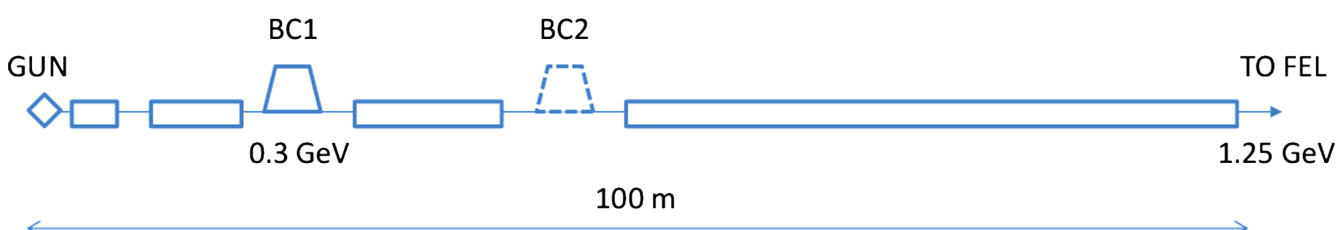

FIG. 1. Sketch of LCLS (top) and FERMI (bottom) beam lines (not in scale).
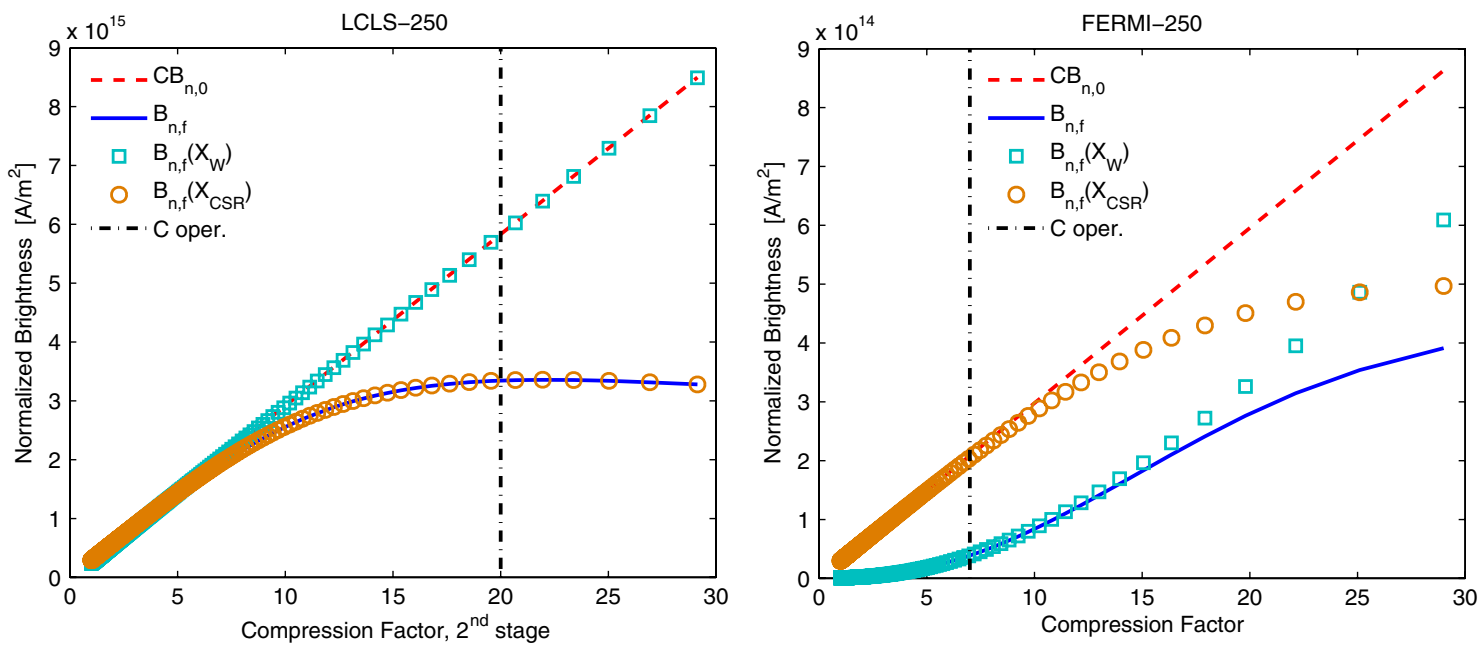

FIG. 2. Theoretical final normalized brightness in LCLS (left) and in FERMI (right) as a function of the compression factor, for $250 \mathrm{pC}$ beam charge. The nominal (unperturbed) brightness is shown with a dashed line, the effective (perturbed) brightness is shown with a solid line, CSR (circles), and GTW dominated brightness (squares) is also shown. The dash-dotted line identifies the operational compression factor.

dependence of the final brightness on these two parameters for FERMI-250, while keeping all other parameters unchanged (see Table I). The values of $\Delta$ and $\bar{\beta}$ used in Table I and in Fig. 3 reflect the design estimates both for LCLS and FERMI, with the only exception of FERMI-500. In this case, the linac misalignment is a guess to match the experimental data. Its smaller value is consistent with the improvement in trajectory control made necessary in order to minimize the GTW effect.
Suppression of GTW effect is thus described in our model with an overall smaller misalignment of the linac with respect to the beam reference trajectory. At the end, also considering that the experimental brightness measurement error is dominated by the emittance measurement error [23] and by the uncertainty on the bunch length [31,33], the analysis is able to predict the effective brightness with an accuracy in the range $10 \%-20 \%$. Of equal importance as the brightness prediction, the model 


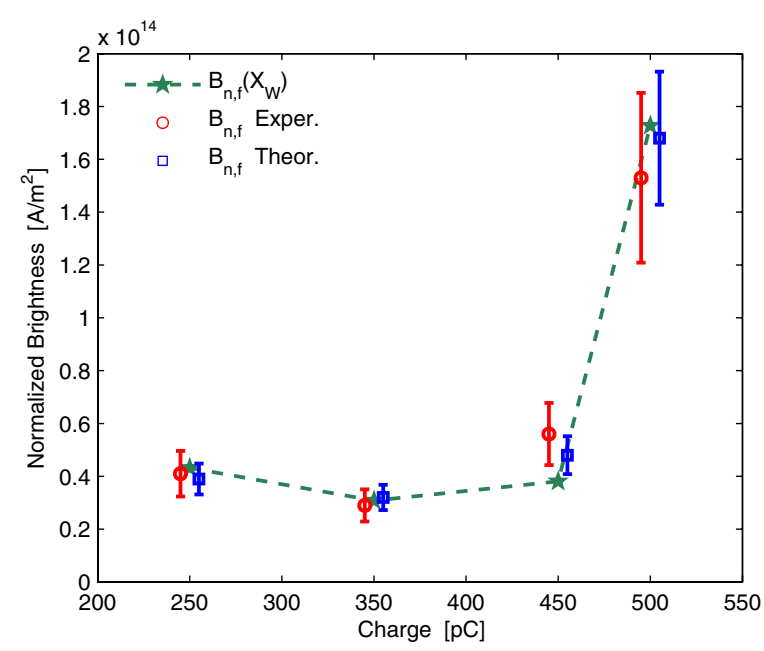

FIG. 3. Measured (circles) and predicted (squares) normalized brightness at the end of the FERMI linac as a function of the beam charge. The compression factors are 7, 6, 6, and 12 for beam charge values of $250,350,450$, and $500 \mathrm{pC}$, respectively. The compression and charge markers are shifted in the abscissa for better reading. The dashed line, which is used to guide the eye, represents Eq. (6) when only GTW is considered.

is able to recognize the dominant degradation source through plots like those of Fig. 2.

\section{BRIGHTNESS OPTIMIZATION}

We now turn our attention to the brightness of beams that were introduced in $[29,37,38]$ : these are usually called "ultralow charge" beams because their charge is smaller than a few tens of pC. This kind of beams is usually associated to electron diffraction applications by virtue of their very small transverse emittance, and to single-spike self-amplified spontaneous emission FELs (see [39-41] and references therein) on account of their femtosecond
TABLE II. Ultralow charge beam and machine parameters. The peak current $I_{0}$ is defined as $Q c$ divided by $3.5 \sigma_{z, 0}$. The normalized emittance is the geometric mean of the horizontal and the vertical values. The $\sim 1 \mathrm{pC}$ bunch length is initially compressed with velocity bunching $(\rightarrow)$ as described in $[37,38]$. The magnetic compression is set to obtain a final peak current of $1.5 \mathrm{kA}$ in all cases.

\begin{tabular}{|c|c|c|c|c|c|}
\hline Parameter & PAL-1 & UCLA-1 & LCLS-20 & FERMI-100 & Units \\
\hline$Q$ & 1.6 & 1.0 & 20 & 100 & $\mathrm{pC}$ \\
\hline$\sigma_{t 0}$ & $1.2 \rightarrow 0.002$ & $1.6 \rightarrow 0.002$ & 1.3 & 1.2 & ps \\
\hline$I_{0}$ & $0.4 \rightarrow 200$ & $0.2 \rightarrow 140$ & 5 & 25 & A \\
\hline C & $1 \times 7$ & $1 \times 11$ & $10 \times 34$ & $5 \times 13$ & $\ldots$ \\
\hline$\gamma_{0}$ & 490 & 490 & 490 & 1470 & $\cdots$ \\
\hline$\gamma_{f}$ & 20550 & 20550 & 20550 & 2450 & $\cdots$ \\
\hline$L_{\mathrm{FODO}}$ & 6,680 & 6,790 & 6,790 & 12,45 & $\mathrm{~m}$ \\
\hline \multicolumn{6}{|l|}{$L_{\mathrm{tot}}$} \\
\hline $\bar{\beta}$ & 35 & 35 & 35 & 25 & $\mathrm{~m}$ \\
\hline$\hat{\beta}$ & 3 & 3 & 3 & 3 & $\mathrm{~m}$ \\
\hline$\Delta$ & 200 & 200 & 200 & 100 & $\mu \mathrm{m}$ \\
\hline$W_{\perp 0}$ & $1 \times 10^{16}$ & $1 \times 10^{16}$ & $1 \times 10^{16}$ & $17 \times 10^{16}$ & $\mathrm{~V} / \mathrm{C} / \mathrm{m}^{2}$ \\
\hline$\varepsilon_{n 0}$ & 0.13 & 0.04 & 0.2 & 0.3 & $\mu \mathrm{m} \mathrm{rad}$ \\
\hline$C B_{n, 0}$ & $8.88 \times 10^{16}$ & $9.41 \times 10^{17}$ & $3.74 \times 10^{16}$ & $1.67 \times 10^{16}$ & $\mathrm{~A} / \mathrm{m}^{2}$ \\
\hline $\begin{array}{l}B_{n, f} \\
\text { (Eq. (6)) }\end{array}$ & $2.47 \times 10^{16}$ & $2.61 \times 10^{17}$ & $2.03 \times 10^{16}$ & $1.11 \times 10^{16}$ & $\mathrm{~A} / \mathrm{m}^{2}$ \\
\hline
\end{tabular}

bunch duration. The beam parameters and predicted brightness are summarized in Table II. Figure 5 (left plot) shows the brightness of ultralow charge beams after having been hypothetically accelerated and time compressed in the LCLS beam delivery system. In all scenarios, the magnetic compression was set for a $1.5 \mathrm{kA}$ final peak current. The CSR wake turns out to be the only important perturbation for these beams since, according to Eqs. (5) and (6), low charge and short duration suppress the GTW instability. A normalized brightness of up to 2 orders of magnitude higher than that currently achieved in existing facilities appears reachable.
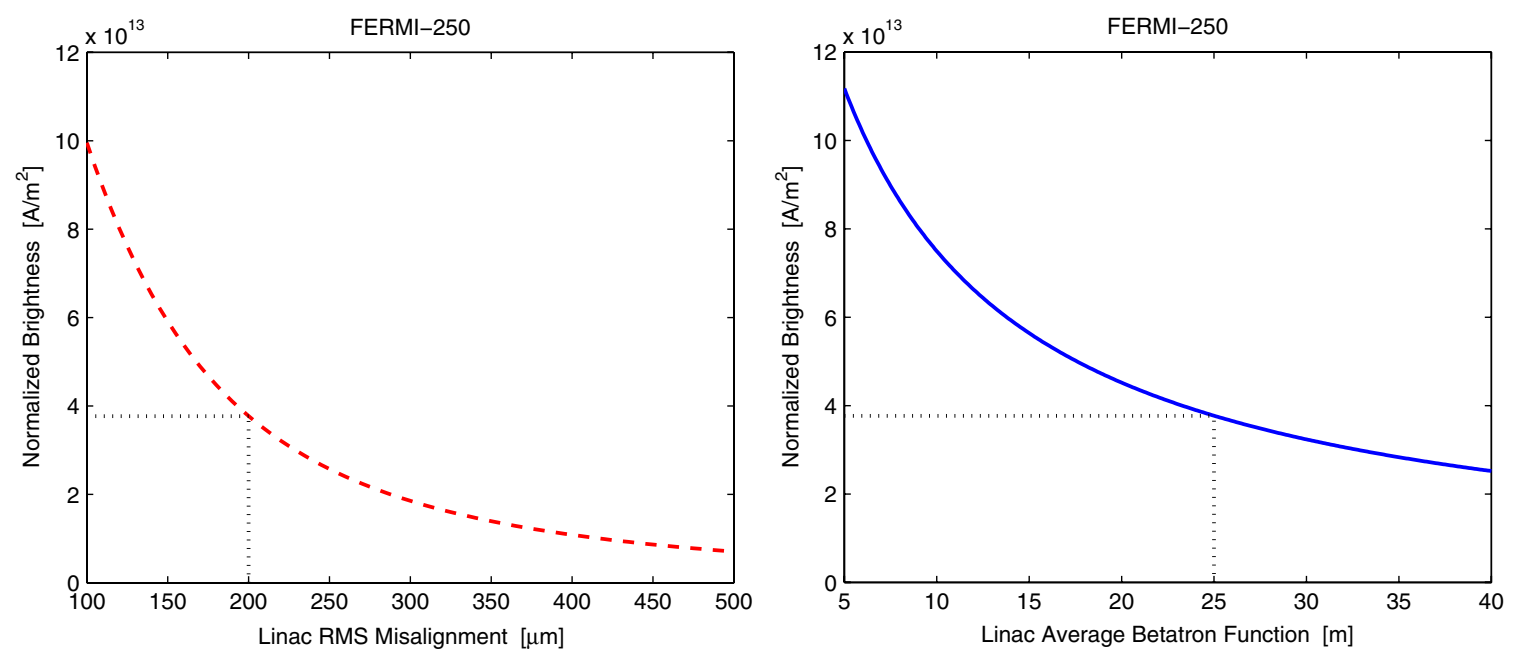

FIG. 4. Final brightness for the FERMI-250 scenario as a function of the linac rms misalignment $\Delta$ (left) and linac average betatron function $\bar{\beta}$ (right). Dotted lines in both plots identify the actual working point (see Table I). 

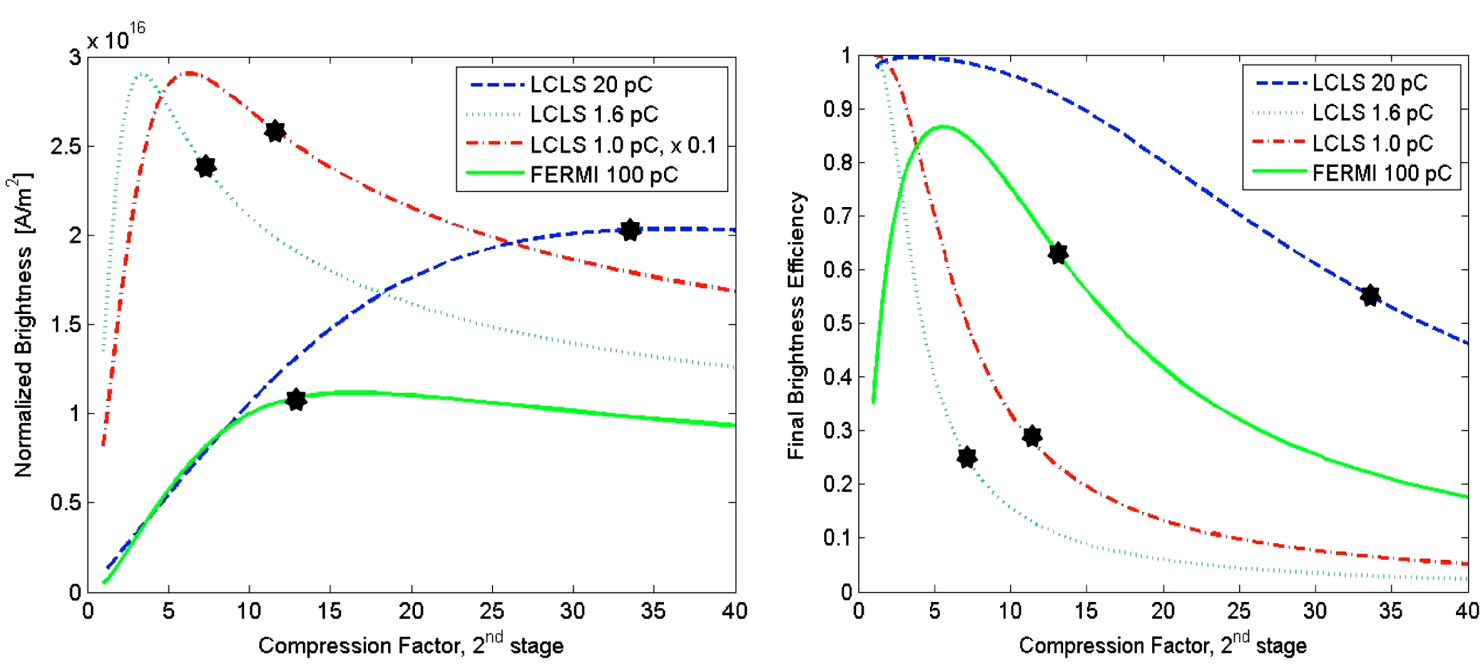

FIG. 5. Final normalized brightness (left) and brightness efficiency (right) as a function of the compression factor in the second compressor, for the scenarios depicted in Table II. The star identifies the compression factor that is needed to reach $1.5 \mathrm{kA}$ final peak current.

The FERMI-100 case is the result of an optimization of the parameters involved in Eq. (6) that tends to maximize the brightness efficiency $B_{n, f} / C B_{n, 0}$, as shown in Fig. 5 (right plot). This optimized scenario improves the current FERMI brightness (see Table I) by a factor $\sim 100$, reaching a level as high as that of the 1.6 and $20 \mathrm{pC}$ beams accelerated in LCLS, for the same final peak current. It is worth noticing that, although the brightness gain factor of 100 is partly provided by higher initial brightness and compression factor, there is no guarantee a priori that GTW and CSR effects, once taken into account, would sustain the same gain. The analysis shows that this is indeed the case, leading to a working point that ensures high brightness efficiency.

\section{CONCLUSIONS}

We have formulated a limit on the electron beam brightness achievable in a linac in the presence of geometric transverse wakefield and coherent synchrotron radiation. The predicted brightness agrees with the experimental value in LCLS and in FERMI, over different working points, once those collective effects are taken into account simultaneously. Of equal importance as the brightness prediction, the model is able to recognize the dominant source of brightness degradation and, thus, reveals as a fast exploratory tool to maximize the beam brightness with a proper choice of the working point.

We then show that a normalized brightness in excess of that currently achieved, can be provided by ultralow charge (1-20 pC), ultrashort beams ( $\sim 1 \mathrm{fs})$ that totally suppress the GTW instability. A normalized brightness in the range $10^{16}-10^{17} \mathrm{~A} / \mathrm{m}^{2}$ is obtainable. The proposed model was used to find out more standard beam parameters $(100 \mathrm{pC}$ totally compressed by a factor 65) for the FERMI FEL that would allow an improvement of the present brightness by 2 orders of magnitude. In this way, a $\sim 10^{16} \mathrm{~A} / \mathrm{m}^{2}$ normalized brightness could be achieved during normal FERMI operations, with expected advantage for the FEL output peak power.

\section{ACKNOWLEDGMENTS}

This work is partially based on the experience that the author collected during the FERMI FEL commissioning. For this reason, the author acknowledges the FERMI Commissioning Team and, in particular, E. Allaria, P. Craievich, G. Penco, and M. Trovo' for the hard work and the support they provided during that activity. The author is grateful to M. Cornacchia for his careful reading of this article and instructive suggestions for improving it. Work supported in part by the Italian Ministry of University and Research under Grants No. FIRBRBAP045JF2 and No. FIRB-RBAP06AWK3.

[1] J. Blau, Y.H. Bae, K. Cohn, W. B. Colson, and J. M. Wittrock, in Proceedings of the 32th Free Electron Laser Conference, Malmö, Sweden (MAX-lab, Sweden, 2010), MOPA05.

[2] J. Ellis and Ian Wilson, Nature (London) 409, 431 (2001).

[3] S. Di Mitri et al., Nucl. Instrum. Methods Phys. Res., Sect. A 608, 19 (2009).

[4] S. Di Mitri, Ph.D. thesis, University of Groningen, The Netherlands, 2011.

[5] M. Reiser, Theory and Design of Charged Particle Beams (Wiley-VCH, Weinheim, 2008).

[6] I. Zagorodnov and M. Dohlus, Phys. Rev. ST Accel. Beams 14, 014403 (2011).

[7] I. V. Bazarov and C. K. Sinclair, Phys. Rev. ST Accel. Beams 8, 034202 (2005). 
[8] R. Bartolini, M. Apollonio, and I. P. S. Martin, Phys. Rev. ST Accel. Beams 15, 030701 (2012).

[9] K. L. F. Bane, Report No. SLAC-PUB-9663, 2003.

[10] P. Craievich, T. Weiland, and I. Zagorodnov, Nucl. Instrum. Methods Phys. Res., Sect. A 558, 58 (2006).

[11] E. L. Saldin, E. A. Schneidmiller, and M. V. Yurkov, Nucl. Instrum. Methods Phys. Res., Sect. A 398, 373 (1997).

[12] P. Emma et al., Nat. Photonics 4, 641 (2010).

[13] E. Allaria et al., Nat. Photonics 6, 699 (2012).

[14] R. Talman, Phys. Rev. Lett. 56, 1429 (1986).

[15] T. Nakazato, M. Oyamada, N. Niimura, S. Urasawa, O. Konno, A. Kagaya, R. Kato, T. Kamiyama, Y. Torizuka, T. Nanba, Y. Kondo, Y. Shibata, K. Ishi, T. Ohsaka, and M. Ikezawa, Phys. Rev. Lett. 63, 1245 (1989).

[16] Ya.S. Derbenev, J. Rossbach, E. L. Saldin, and V.D. Shiltsev, Report No. TESLA-FEL 95-05, 1995.

[17] H. Braun, F. Chautard, R. Corsini, T. O. Raubenheimer, and P. Tenenbaum, Phys. Rev. Lett. 84, 658 (2000).

[18] H. H. Braun, R. Corsini, L. Groening, F. Zhou, A. Kabel, T. O. Raubenheimer, R. Li, and T. Limberg, Phys. Rev. ST Accel. Beams 3, 124402 (2000).

[19] F. Stulle, A. Adelmann, and M. Pedrozzi, Phys. Rev. ST Accel. Beams 10, 031001 (2007).

[20] R. Talman, N. Malitsky, and F. Stulle, Phys. Rev. ST Accel. Beams 12, 014201 (2009).

[21] S. Di Mitri, M. Cornacchia, and S. Spampinati, Phys. Rev. Lett. 110, 014801 (2013).

[22] E. D. Courant and H.S. Snyder, Ann. Phys. (Paris) 3, 1 (1958).

[23] S. Di Mitri, E. M. Allaria, P. Craievich, W. Fawley, L. Giannessi, A. Lutman, G. Penco, S. Spampinati, and M. Trovo', Phys. Rev. ST Accel. Beams 15, 020701 (2012).

[24] A. W. Chao, Physics of Collective Beam Instabilities in High Energy Accelerators (Wiley, New York, 1993), ISBN 0-471-55184-8.

[25] G. Guignard and J. Hagel, Nucl. Instrum. Methods Phys. Res., Sect. A 434, 179 (1999).

[26] T. O. Raubenheimer, Phys. Rev. ST Accel. Beams 3, 121002 (2000).
[27] P. Craievich, S. Di Mitri, and A. A. Zholents, Nucl. Instrum. Methods Phys. Res., Sect. A 604, 457 (2009).

[28] I. V. Bazarov, B. M. Dunham, and C. K. Sinclair, Phys. Rev. Lett. 102, 104801 (2009).

[29] Y. Ding et al., Phys. Rev. Lett. 102, 254801 (2009).

[30] J. Arthur et al., LCLS Conceptual Design Report No. SLAC-R-593, 2002 [http://www-ssrl.slac.stanford .edu/lcls/cdr/].

[31] S. Di Mitri, E. Allaria, R. Appio, L. Badano, G. De Ninno, D. Castronovo, M. Cornacchia, P. Craievich, S. Ferry, L. Fröhlich, A. Lutman, G. Penco, C. Scafuri, S. Spampinati, C. Spezzani, M. Trovò, M. Veronese, and S. V. Milton, in Proceedings of the 32th Free Electron Laser Conference, Malmo, Sweden (Ref. [1]), MOPA02.

[32] C. Bocchetta et al., FERMI@Elettra Conceptual Design Report No. ST/F-TN-07/12, 2007 [https://www.elettra .trieste.it/FERMI/index.php?n=Main.CDRdocument].

[33] S. Di Mitri et al., in Proceedings of the 2nd International Particle Accelerator Conference, San Sebastián, Spain (EPS-AG, Spain, 2011), TUZA01.

[34] F. Wang, C. Adolphsen, and C. Nantista, Phys. Rev. ST Accel. Beams 14, 010401 (2011).

[35] R. Bartolini, Nucl. Instrum. Methods Phys. Res., Sect. A 657, 177 (2011).

[36] Y. Sun, C. Adolphsen, C. Limborg-Deprey, T. Raubenheimer, and J. Wu, Phys. Rev. ST Accel. Beams 15, 030703 (2012).

[37] J.-H-Han, Phys. Rev. ST Accel. Beams 14, 050101 (2011).

[38] R. K. Li, K. G. Roberts, C. M. Scoby, H. To, and P. Musumeci, Phys. Rev. ST Accel. Beams 15, 090702 (2012).

[39] J. B. Rosenzweig et al., Nucl. Instrum. Methods Phys. Res., Sect. A 593, 39 (2008).

[40] S. Reiche, P. Musumeci, C. Pellegrini, and J. B. Rosenzweig, Nucl. Instrum. Methods Phys. Res., Sect. A 593, 45 (2008).

[41] M. Boscolo, M. Ferrario, I. Boscolo, F. Castelli, S. Cialdi, V. Petrillo, R. Bonifacio, L. Palumbo, and L. Serafini, Nucl. Instrum. Methods Phys. Res., Sect. A 593, 137 (2008). 